\title{
A highly sensitive dual-read assay using nitrogen-doped carbon dots for the quantitation of uric acid in human serum and urine samples
}

\author{
Fan $\mathrm{Li}^{1} \cdot$ Jiahan Rui ${ }^{1} \cdot$ Ziyu Yan ${ }^{1} \cdot$ Ping Qiu ${ }^{1,2}$ (D) Xiaomin Tang ${ }^{3}$ \\ Received: 9 May 2021 / Accepted: 2 August 2021 / Published online: 29 August 2021 \\ (C) The Author(s), under exclusive licence to Springer-Verlag GmbH Austria, part of Springer Nature 2021
}

\begin{abstract}
A simple dual-read assay for uric acid (UA) was developed based on a combined ratiometric fluorescent and colorimetric strategy using nitrogen-doped carbon dots (N-CDs). The biosensor relies on the oxidation of UA by uricase to produce $\mathrm{H}_{2} \mathrm{O}_{2}$, which was then converted to ${ }^{\circ} \mathrm{OH}$ radicals by $\mathrm{I}^{-}$, resulting in the oxidation of $o$-phenylenediamine (OPD) to 2,3-diaminophenazine (DAP). In the presence of UA, the colorless biosensor system changed to yellow. Furthermore, the presence of DAP quenched the fluorescence emission of the N-CDs at $427 \mathrm{~nm}$ based on the inner filter effect (IFE). With increasing UA concentrations, the fluorescence intensity of the biosensor at $427 \mathrm{~nm}$ decreased but increased at $580 \mathrm{~nm}$, demonstrating the ratiometric response. A strong linearity was observed between the fluorescence intensity ratio of DAP to N-CDs $\left(I_{580} / I_{427}\right)$ and the corresponding UA concentration over the range $0.5-150 \mu \mathrm{M}$, and a limit of detection $(\mathrm{S} / \mathrm{N}$ ratio of 3$)$ of $0.06 \mu \mathrm{M}$ was calculated. The dual-read assay was successfully employed in the quantitation of UA in human serum and urine samples, revealing its potential for measuring UA in clinical samples.
\end{abstract}

Keywords Uric acid $\cdot$ Dual-mode detection $\cdot$ Nitrogen-doped carbon dots $\cdot$ O-Phenylenediamine $\cdot$ Iodide-peroxidase-like activity $\cdot$ Serum and urine samples

\section{Introduction}

Uric acid (UA) is one of the final metabolites of purine metabolism in the human body. It is unable to undergo further oxidation to 5-hydroxyisourate because of the lack of urate oxidase in humans [1]. Under normal physiological conditions, the concentration of UA ranges from 120 to $460 \mu \mathrm{M}$ in blood serum and 1.4 to $4.4 \mathrm{mM}$ in urinary excretion $[2,3]$. Maintaining a normal level of UA in bodily fluids is vital for ensuring proper health because changes in the concentration of UA can cause or reflect a host of diseases. Abnormal levels of UA can increase the risk of cardiovascular disease, gout, primary hypertension, kidney diseases, and multiple sclerosis

Ping Qiu

pingqiu@ncu.edu.cn

1 Department of Chemistry, Nanchang University, Nanchang 330031, China

2 Jiangxi Province Key Laboratory of Modern Analytical Science, Nanchang University, Nanchang 330031, China

3 The Fourth Affiliated Hospital, Nanchang University, Nanchang 330003, China
[4-8]. It has also been reported that patients infected with COVID-19 (coronavirus disease 2019) commonly develop renal complications, which are reflected in their UA, blood urea, serum creatinine, and D-dimer levels [9]. Recent studies suggest that measuring UA levels may be helpful in screening patients suspected of COVID-19 [10]. Thus, a method that enables the rapid and sensitive detection of UA in blood serum and urine samples would be quite profound for the early detection of various human diseases.

To date, various techniques have been used for the quantitative analysis of UA, such as high-performance liquid chromatography (HPLC) [11], fluorescence spectroscopy [12], electrochemistry [13], ultraviolet (UV) absorption spectroscopy [14], colorimetric assays [15], chemiluminescence [16], and surface-enhanced Raman spectroscopy [17]. However, these techniques only allow for the detection of a single signal. Compared to a single-signal output, dual-signal readouts are more beneficial for reducing instrumental and operational interferences [18]. At present, spectroscopic analysis of UA has attracted widespread attention because of its high sensitivity and rapid and simplistic operation. Among them, ratiometric fluorescence spectroscopy provides much more reliable quantitative results [19] than other absorbance and fluorescence spectroscopic techniques because ratiometric fluorescence 
analysis can effectively normalize any variation in fluorescence readouts by dividing the readouts of two different optical signals. In addition, the change in color of the fluorescent indicator in response to the analyte can be visually observed, which makes colorimetric methods popular in applications that necessitate rapid determination. Therefore, ratiometric fluorescence spectroscopy coupled with colorimetric detection of UA was proposed.

Carbon dots (CDs) are an emerging class of carbon-based nanomaterials that exhibit high intrinsic fluorescence brightness and low toxicity [20]. At present, CDs have been successfully utilized as nanoprobes [21] and drug delivery vehicles [22], as well as in bioimaging [23] and optoelectronic conversion [24]. Doping has been a sought-after strategy for controlling the properties of nanomaterials, including carbon dots [25]. Doping CDs with molecules containing amine functionalities has enabled surface passivation. Because nitrogen atoms have suitable atomic radii and five valence electrons, they bond easily with carbon atoms [26]. It is an approach that can improve the optical and electrical properties of CDs.

Herein, we constructed a ratiometric fluorescent and colorimetric sensor based on nitrogen-doped carbon dots $(\mathrm{N}-$ CDs) for the selective detection of UA in solution. In the sensing assay, iodide was used as a replacement for the conventional horseradish peroxidase used in colorimetric assays because it is a common and inexpensive reagent and exhibits peroxidase-like activity [27, 28], which makes it an ideal peroxidase mimetic. Catalyzed by uricase, UA generated $\mathrm{H}_{2} \mathrm{O}_{2}$ after being oxidized to 5-hydroxyisourate [29], which was converted to hydroxyl radicals ( $(\mathrm{OH})$ in the presence of iodide ions. The ${ }^{\circ} \mathrm{OH}$ species then oxidized $o$-phenylenediamine (OPD) to produce 2,3-diaminophenazine (DAP), which produced yellow fluorescence emission at $580 \mathrm{~nm}$ and quenched the fluorescence emission of the N-CDs at $427 \mathrm{~nm}$ based on the inner filter effect (IFE). Therefore, when the concentration of UA increased, the fluorescence intensity of the N-CDs at $427 \mathrm{~nm}$ quenched, while the fluorescence at $580 \mathrm{~nm}$ increased. Based on this principle, the ratio of the fluorescence intensity of DAP to N-CDs $\left(I_{580} / I_{427}\right)$ was used for the quantitative analysis of UA. In addition, as the concentration of UA increased, the color of the solution containing the biosensor system gradually changed to yellow. Furthermore, the dualread assay for the quantitation of UA was ultra-sensitive and highly selective and was successfully employed for the quantitation of UA in human serum and urine samples.

\section{Experimental}

\section{Chemicals and materials}

Uric acid, uricase, urea, glucose, creatinine, dopamine, and glutathione were purchased from Sigma-Aldrich (St. Louis,
$\mathrm{MO}$ ), while $o$-phenylenediamine (OPD), histidine, and phenylalanine were obtained from Macklin Biochemical Technology Co., Ltd. (Shanghai, China). Diethylenetriamine (DETA) was obtained from TCI Shanghai Chemical Industry Development Co., Ltd. (Shanghai, China). All chemicals used were of analytical grade. The ultrapure water $(18.25 \mathrm{M} \Omega$ $\mathrm{cm}^{-1}$ ) was used in all experiments and was prepared from a Milli-Q water purification system (Millipore, USA).

\section{Measurement and apparatus}

All fluorescence spectra were acquired by an F-4600 fluorescence spectrometer (Hitachi, Japan) with an excitation wavelength of $330 \mathrm{~nm}$. UV-Vis absorption spectra were acquired on an Agilent Cary 8454 UV-Vis spectrophotometer (Agilent Technologies, USA). Fluorescence decay curves were acquired on a photoluminescence spectrometer (Edinburgh FLS1000, UK). The transmission electron microscopy (TEM) was used to study the morphology of the N-CDs using a JEM-2100F microscope (JEOL Co., Japan). X-ray photoelectron spectroscopy (XPS) was conducted to analyze the elemental composition of the N-CDs using an Esca Lab $250 \mathrm{Xi}$ instrument with $\mathrm{Al} \mathrm{K} \alpha$ radiation as the excitation source. Fourier transform infrared (FT-IR) spectra were acquired on a Shimadzu IR Prestige-21 spectrometer in transmission mode by preparing $\mathrm{KBr}$ pellets with the N-CDs. Zeta potentials were obtained on a Nano ZS90 nanoparticle analyzer (Malvern, UK). The biochemical analysis of the UAcontaining samples was conducted with a biochemical analyzer (AU2700, Beckman, Olympus, USA).

\section{Synthesis of the N-CDs}

The N-CDs were prepared by hydrothermal synthesis according to a previously published procedure [30] with modifications. First, citric acid monohydrate (1.2 g) was dissolved in $20 \mathrm{~mL}$ of ultra-pure water. The solution was supplemented with DETA $(0.15 \mathrm{~mL})$ and sonicated for $15 \mathrm{~min}$. The mixture was transferred to a $50-\mathrm{mL}$ Teflon-lined stainless steel autoclave and was heated at $200^{\circ} \mathrm{C}$ for $14 \mathrm{~h}$. The resulting brownyellow product was dialyzed through a dialysis membrane for more than 3 days. Finally, the powdered N-CDs were obtained by freeze-drying and stored at room temperature until further use.

\section{Procedure for UA analysis}

Aliquots $(50 \mu \mathrm{L})$ of standard solutions of UA with different concentrations and $50 \mu \mathrm{L}$ uricase $\left(100 \mu \mathrm{g} \cdot \mathrm{mL}^{-1}\right)$ were successively pipetted into a solution $(100 \mu \mathrm{L})$ of BrittonRobinson buffer ( $\mathrm{pH}$ 8.5). The mixture was stabilized for $15 \mathrm{~min}$ at $37^{\circ} \mathrm{C}$ and then supplemented successively with $200 \mu \mathrm{L}$ OPD (25 mM), $200 \mu \mathrm{L} \mathrm{I}^{-}(14 \mathrm{mM}), 60 \mu \mathrm{L}$ N-CDs 
(400 $\mu \mathrm{g} \cdot \mathrm{mL}^{-1}$ ), and $1340 \mu \mathrm{L}$ phosphate buffer saline (PBS, $10 \mathrm{mM}, \mathrm{pH}$ 5.5). The resulting solution was incubated at $45^{\circ} \mathrm{C}$ for another $30 \mathrm{~min}$. Absorbance spectra of the solution were recorded by a UV-Vis spectrophotometer, and the fluorescence spectra were acquired after excitation at $330 \mathrm{~nm}$ with the slit width of the excitation and emission monochromators at $5.0 \mathrm{~nm}$.

\section{Analysis of UA in human serum and urine samples}

To demonstrate the application of the proposed method, the ratiometric fluorescent system comprising the N-CDs was utilized to quantitatively analyze UA in human serum and urine samples. Human blood samples provided by the Fourth Affiliated Hospital of Nanchang University (Nanchang, China) were centrifuged at $4000 \mathrm{rpm}$ for $15 \mathrm{~min}$, after which the supernatants (serum) were extracted and diluted 5-fold using PBS solution. To verify the results of the N-CDsbased fluorescence system, the human serum samples were also analyzed by an Olympus AU2700 automatic biochemical analyzer. Before biochemical analysis, the serum samples were centrifuged at $4000 \mathrm{rpm}$ for $3 \mathrm{~min}$. Each of the resulting supernatants $(5.6 \mu \mathrm{L})$ was supplemented with aliquots of uricase, 4-aminoantipyrine (4-AAP), and 3diphenylamine disodium salt (MADB). After that, the mixtures were analyzed by the biochemical analyzer at $660 / 800$ nm.

The urine samples were collected from two volunteers in the morning and filtered through a $0.22-\mu \mathrm{m}$ Millipore filter. The resulting filtrates were centrifuged at $8000 \mathrm{rpm}$ for $5 \mathrm{~min}$ and diluted 50-fold with PBS solution for further use.

\section{Results and discussion}

\section{Characterization of N-CDs}

The morphology and size of the synthesized N-CDs were analyzed by TEM. As showed in Fig. 1a, the TEM images revealed that the N-CDs had a uniformly spherical shape and presented excellent dispersion in aqueous solution. Additionally, the particles had an average diameter of about $2.8 \mathrm{~nm}$ (Fig. 1b). In the high-resolution TEM image (Fig. 1a, inset), common ordered lattices of the N-CDs were observed with a lattice spacing of $0.21 \mathrm{~nm}$, which corresponded to the (100) crystal face of the graphitic carbon [31]. The structure of the N-CDs was also characterized by FT-IR (Fig. 1c). The FTIR spectrum featured a broad absorption band at $3429 \mathrm{~cm}^{-1}$, which corresponded to the $-\mathrm{OH}$ stretching vibration. Furthermore, the peak located around $2900 \mathrm{~cm}^{-1}$ was attributed to $\mathrm{C}-\mathrm{H}$ alkyl stretching vibration. The absorption band at $1681 \mathrm{~cm}^{-1}$ was attributed to $\mathrm{C}=\mathrm{O}$ groups on the surface of the $\mathrm{N}-\mathrm{CD}$. In addition, the absorption bands at $1580 \mathrm{~cm}^{-1}, 1425$ $\mathrm{cm}^{-1}$, and $1028 \mathrm{~cm}^{-1}$ corresponded to the $\mathrm{C}=\mathrm{C}, \mathrm{C}-\mathrm{N}$, and $\mathrm{C}-\mathrm{O}$ stretching vibrations, respectively. The results showed that not only were there carboxyl group and hydroxyl groups, but also an amino group was on the surface of N-CDs. To further corroborate the formation of the N-CDs, XPS was conducted. The full-scan survey spectrum of the N-CDs (Fig. 1d) featured $\mathrm{C} 1 \mathrm{~s}(286.0 \mathrm{eV}), \mathrm{N} 1 \mathrm{~s}(401.5 \mathrm{eV})$, and $\mathrm{O} 1 \mathrm{~s}(534.0$ $\mathrm{eV}$ ) peaks, which confirmed the existence of $\mathrm{C}, \mathrm{N}$, and $\mathrm{O}$ elements in the N-CDs. According to the high-resolution C $1 \mathrm{~s}$ spectrum (Fig. 1e), the binding energies of 289.6, 288.0, 285.7, and $284.5 \mathrm{eV}$ corresponded to $\mathrm{C}=\mathrm{O}, \mathrm{C}-\mathrm{O}$, $\mathrm{C}-\mathrm{N}$, and $\mathrm{C}-\mathrm{C}$ bonds, respectively [32]. It further indicated that nitrogen atoms have been successfully doped into CDs. The N 1s core level spectrum (Fig. 1f) featured two separate peaks at $399.2 \mathrm{eV}$ and $400.3 \mathrm{eV}$ that were attributed to pyrrolic $\mathrm{N}$ and graphite $\mathrm{N}$, respectively [33]. These results further substantiated the successful synthesis of the N-CDs.

\section{Optical properties of $\mathrm{N}-\mathrm{CDs}$}

Figure 2a displays the UV-Vis absorption spectrum of the NCDs. There were two significant absorption peaks at $277 \mathrm{~nm}$ and $321 \mathrm{~nm}$ that were associated with the $\pi-\pi^{*}$ transition of the $\mathrm{sp}^{2}$ carbon in N-CDs and $\mathrm{n}-\pi^{*}$ transition of the $\mathrm{C}=\mathrm{O}$ band, respectively [32]. As shown in the fluorescence emission spectra, the emission peak of the N-CDs changed based on the excitation wavelength (Fig. 2b). This phenomenon was caused by new energy levels in the band gaps [30]; as the excitation wavelength increased from 290 to $360 \mathrm{~nm}$, the emission peak red-shifted. The most intense fluorescence emission occurred at a maximum of $430 \mathrm{~nm}$ upon excitation at $330 \mathrm{~nm}$. Moreover, the N-CDs demonstrated excellent fluorescence stability. As shown in Fig. 2c, the fluorescence intensity of the N-CDs did not significantly change over a 14day period while being stored in various solutions of PBS at different $\mathrm{pHs}$ (4.7-7.4). The N-CDs also exhibited a quantum yield of $17.4 \%$, which was determined by measurements using a photoluminescence spectrometer equipped with an integrating sphere.

\section{Choice and function of the materials}

In order to establish a ratiometric fluorescence spectroscopy coupled with colorimetry for the detection of UA, we set our sights on a typical color reaction - catalytic oxidization of OPD (colorless) by $\mathrm{H}_{2} \mathrm{O}_{2}$ and horseradish peroxidase to produce DAP (yellow). Interestingly, OPD has no fluorescence while DAP has strong fluorescence. Thus, this reaction can establish a bridge between fluorescence spectroscopy and colorimetry, which is the first reason to choose OPD. However, DAP produced only one optical signal in the fluorescence spectrum. It required another material to 
Fig. 1 a TEM image of N-CDs, inset: HRTEM image of N-CDs, b the particle size distribution of $\mathrm{N}-\mathrm{CD}$, $\mathbf{c}$ FTIR spectra of N-CDs, d XPS survey spectrum of NCDs, e high-resolution XPS spectra of $\mathrm{C} 1 \mathrm{~s}$, and $\mathbf{f} \mathrm{N} 1 \mathrm{~s}$ peaks of $\mathrm{N}-\mathrm{CDs}$
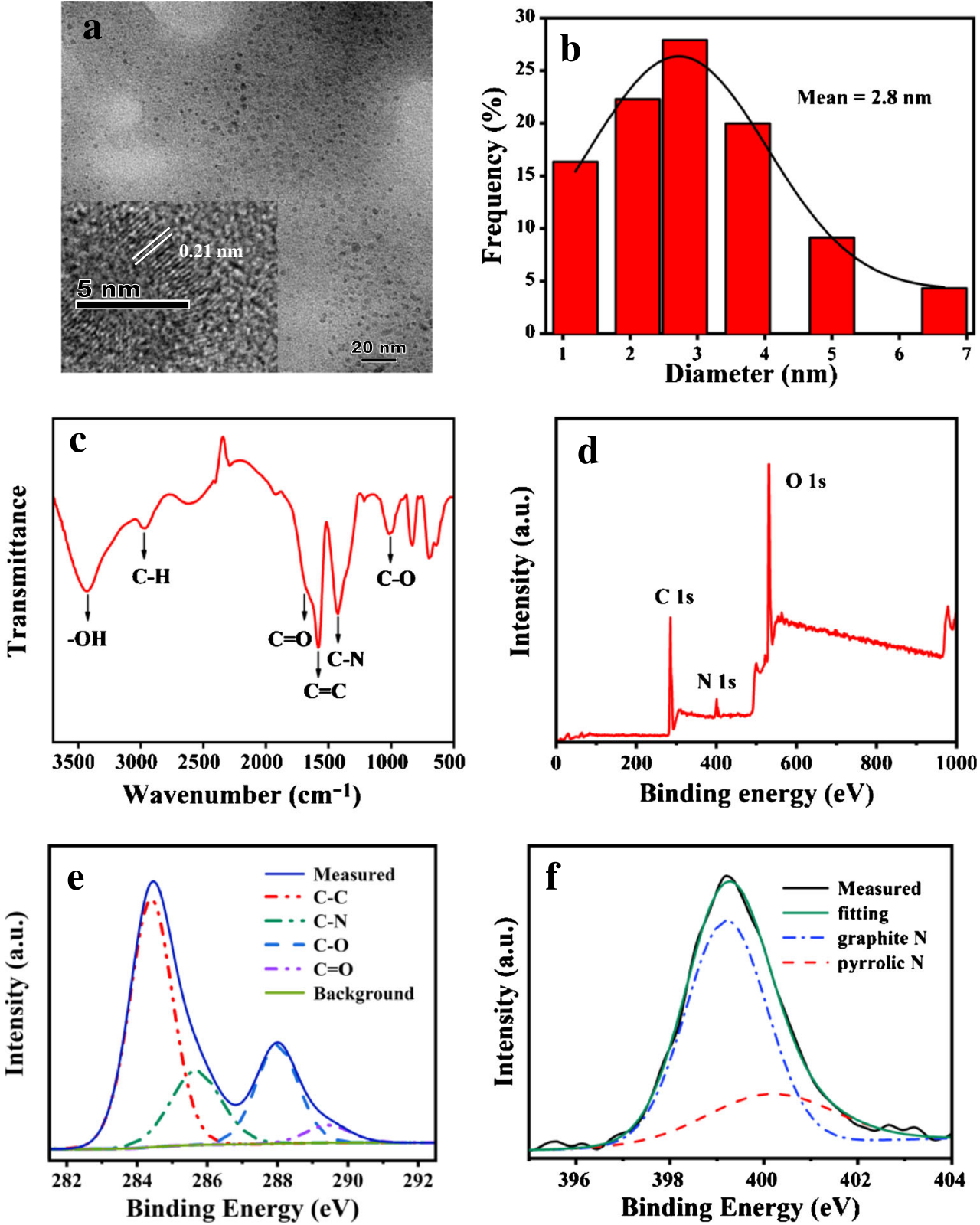

provide the fluorescent optical signal to establish the ratio strategy by the connection between the two optical signals. $\mathrm{N}$-CDs exhibited excellent optical properties (the "Optical properties of N-CDs" section) and can be effectively quenched by DAP. In addition, iodide ions not only showed peroxidase-like activity, but also were inexpensive and common compared with horseradish peroxidase. So, we selected iodide ions to replace horseradish peroxidase.

Scheme 1 illustrates the principle of the dual-read assay for quantifying UA using N-CDs by exploiting the catalytic activity of $\mathrm{I}^{-}$and uricase. Initially, $\mathrm{H}_{2} \mathrm{O}_{2}$ was generated by the oxidation of UA to 5-hydroxyisourate. Furthermore, OPD (colorless) was catalytically oxidized to DAP (yellow color) by $\mathrm{I}^{-}$in the presence of $\mathrm{H}_{2} \mathrm{O}_{2}$. At an excitation wavelength of $330 \mathrm{~nm}$, DAP generated by this reaction quenched the fluorescence of the N-CDs in the system at $427 \mathrm{~nm}$, while the fluorescence intensity at $580 \mathrm{~nm}$ increased. Therefore, both the fluorescence emission and UV-Vis absorption intensities varied with different concentrations of UA. Because of this, the concentration of UA was calculated from the ratio of the fluorescence intensity of DAP/N-CDs $\left(I_{580} / I_{427}\right)$ and the absorbance of DAP at $427 \mathrm{~nm}$.

To elucidate the mechanism of fluorescence quenching of the N-CDs by DAP, several spectroscopic measurements were conducted. As depicted in Fig. 3a, the region around $427 \mathrm{~nm}$ in the absorption spectrum of DAP featured a large spectral 
Fig. 2 a UV-Vis absorption spectroscopy of N-CDs solution $\left(400 \mu \mathrm{g} \mathrm{mL}^{-1}\right)$, b fluorescence emission spectra of N-CDs with the excitation wavelength from 290 to $360 \mathrm{~nm}$, c the fluorescence intensity ratio $\left(I_{580} / I_{427}\right)$ in 14 days of N-CDs phosphate buffer solutions at different $\mathrm{pH}$ values.
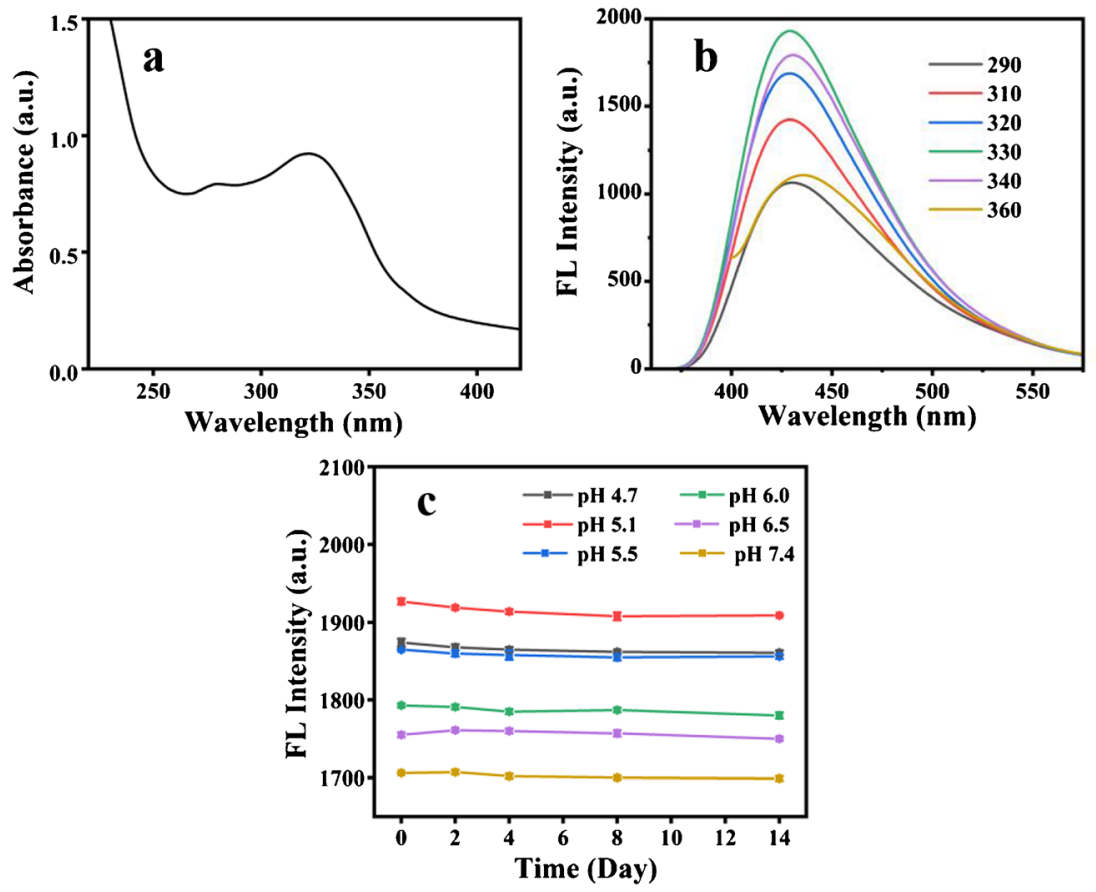

overlap between not only the emission spectra of N-CDs at $427 \mathrm{~nm}$ but also the excitation spectra at $330 \mathrm{~nm}$, which provided evidence for the inner filter effect (IFE) [34]. To further support this mechanism, we measured the fluorescence lifetimes of the N-CDs in the presence and absence of DAP. Interestingly, there was no significant change in fluorescence lifetime of the N-CDs (Fig. 3b), revealing that energy transfer did not occur between the N-CDs and DAP. In addition, the zeta potentials of DAP and N-CDs were $-1.1 \mathrm{mV}$ and -2.6 $\mathrm{mV}$, respectively (Fig. 3c). Because both zeta potentials were negative, it was suggested that there were no electrostatic interactions between DAP and the N-CDs. Moreover, because electrostatic repulsion occurs between identical charges, the distance between N-CDs and DAP tended to be greater than $10 \mathrm{~nm}$. However, the distance between the fluorescence donor and acceptor of should be no more than $10 \mathrm{~nm}$ to enable efficient Förster resonance energy transfer (FRET) [33]. Therefore, the above results revealed that DAP quenched the fluorescence emission of the N-CDs based on IFE.

\section{Feasibility study}

We also investigated the feasibility of the proposed dual-read assay for quantitating UA. As shown in Fig. 4, the fluorescence and UV-Vis spectra of the N-CDs, N-CDs-OPD, NCDs-uricase-OPD-I', N-CDs-UA-uricase-OPD, N-CDsuricase-UA, N-CDs-OPD-I', N-CDs-UA-uricase- $\mathrm{I}^{-}$, and NCDs-UA-uricase-OPD-I' reaction systems were compared. Based on the spectrum in Fig. 4a, only the five major components (N-CDs, UA, uricase, OPD, and $\mathrm{I}^{-}$) coexisted in the system, the fluorescence intensity at $427 \mathrm{~nm}$ would be reduced, and the new emission peak could appear. Thus, each

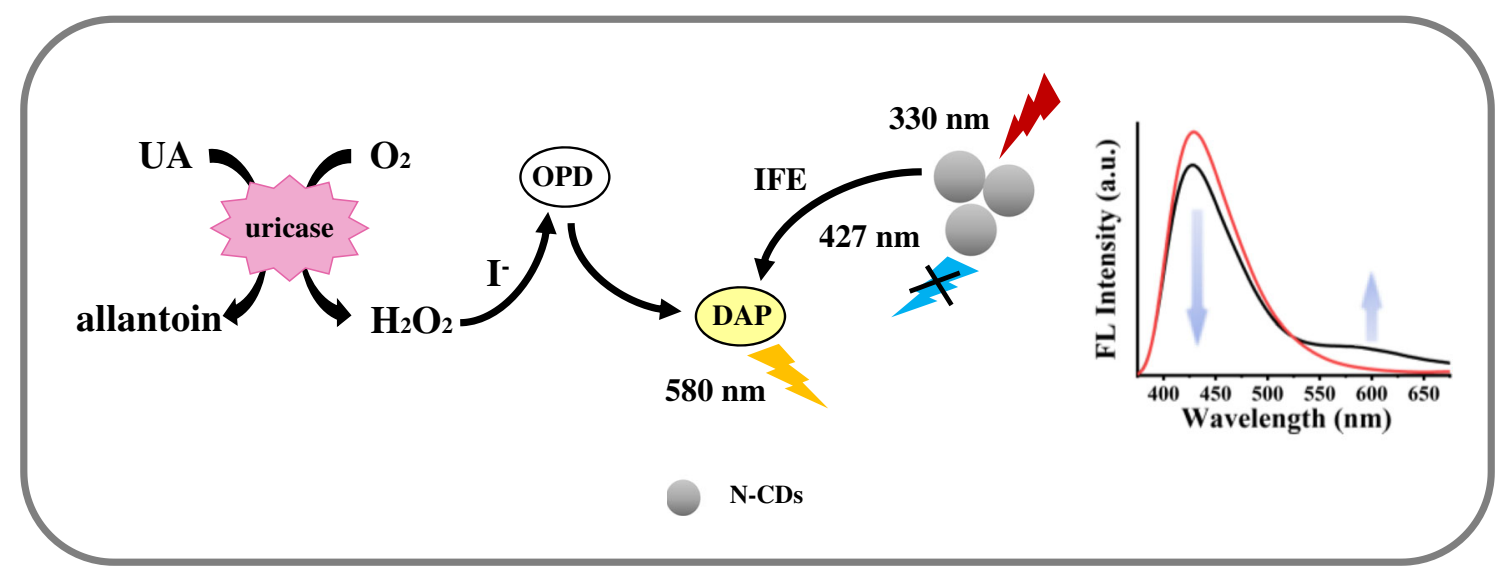

Scheme 1 Schematic illustration of colorimetric and ratiometric fluorescence assay for UA using N-CDs based on $\mathrm{I}^{-}$and uricase. 

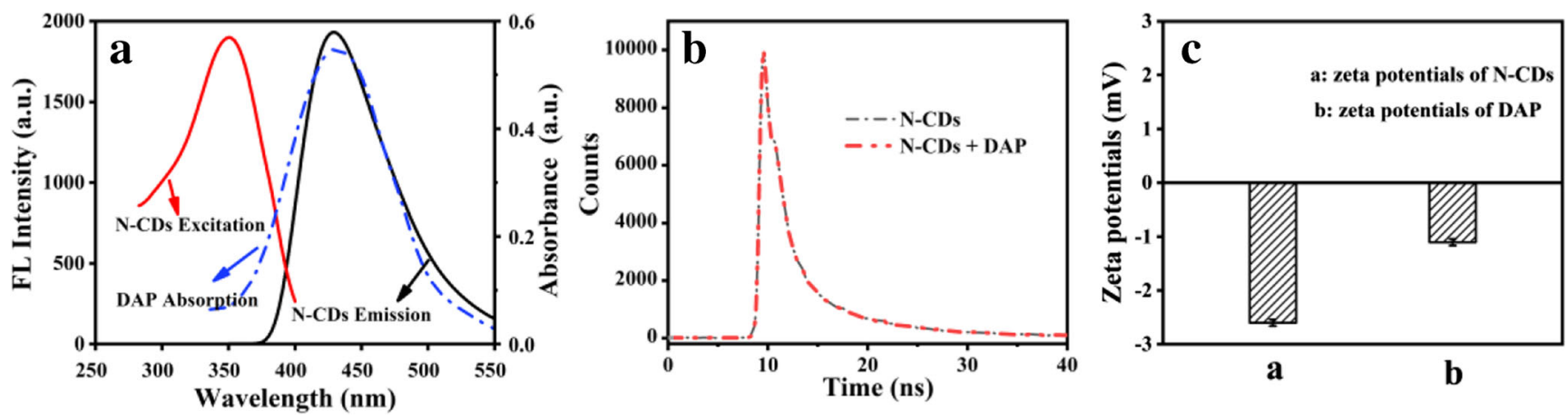

Fig. 3 a UV-Vis absorption spectrum of DAP (blue curve), emission (black curve), and excitation (red curve) spectrum of N-CDs, b the fluorescence lifetime pattern of N-CDs and the mixture of N-CDs and DAP, $\mathbf{c}$ the zeta potentials of N-CDs and DAP

of these substances played a vital role in achieving the quantitation of UA, but their individual presence did not affect the final results. Only the absorption spectrum of the N-CDs-UAuricase-OPD-I' system featured a strong absorption peak with a maximum at $427 \mathrm{~nm}$, which was consistent with the maximum absorption maximum of DAP (Fig. 4b). This indicated that not only was OPD not catalytically oxidized to DAP in other systems but also that iodide ions demonstrated peroxidase-like activity by catalyzing the conversion of $\mathrm{H}_{2} \mathrm{O}_{2}$ to ${ }^{\circ} \mathrm{OH}$. Moreover, the absorbance spectrum of the N$\mathrm{CDs}$ featured no absorption peak near $427 \mathrm{~nm}$. In addition, the $\mathrm{N}-\mathrm{CD}$ s existed only in trace amounts and had no obvious effect on the detection of UA by UV-Vis spectroscopy. Therefore, the dual-read sensor was demonstrated to be successfully applicable for the quantitation of UA.

\section{Optimization of the experimental conditions}

Optimizing the relevant reaction conditions is an important process for improving the sensitivity of the proposed sensor. According to previous work, the catalysis of UA by uricase was most efficient while incubating at $\mathrm{pH} 8.5$ and $35^{\circ} \mathrm{C}$ for $15 \min [35,36]$. To determine the optimal reaction conditions for enabling the most efficient quantitation of UA in biological samples, the influence of $\mathrm{pH}$, temperature, incubation time, $\mathrm{I}^{-}$ concentration, and OPD concentration on the fluorescence intensity ratio of DAP/N-CDs $\left(I_{580} / I_{427}\right)$ was investigated. Based on the results from assessing the influence of $\mathrm{pH}$, the maximum intensity ratio $\left(I_{580} / I_{427}\right)$ was achieved at $\mathrm{pH} 5.5$ (Fig. 5a). Therefore, a $\mathrm{pH}$ of 5.5 was used for subsequent optimization experiments. Next, temperature of the incubation was varied from 25 to $55^{\circ} \mathrm{C}$. Based on the calculated intensity ratios $\left(I_{580} / I_{427}\right)$, the ideal temperature was determined to be $45^{\circ} \mathrm{C}$ (Fig. 5b). Furthermore, the optimum incubation time was found to be $45 \mathrm{~min}$ (Fig. 5c). After varying the concentration of $\mathrm{I}^{-}$from 0.2 to $1.6 \mathrm{mM}$ (Fig. 5d) and the OPD concentration from 0.1 to $3 \mathrm{mM}$ (Fig. 5e), maximum intensity ratios were obtained at $1.4 \mathrm{mM} \mathrm{I}^{-}$and $2.5 \mathrm{mM}$ OPD.

\section{Dual-read assay for UA quantitation}

In order to demonstrate the performance of the biosensor, linear range and limit of detection experiments were conducted to obtain a performance reference for the quantitation of UA based on the optimal conditions described above. As the concentration of UA increased, the emission intensity at $427 \mathrm{~nm}\left(I_{427}\right)$ of the biosensor system at $427 \mathrm{~nm}$ progressively decreased, while the emission intensity at $580 \mathrm{~nm}\left(I_{580}\right)$ increased. In addition, the color of the fluorescence shifted from blue to yellow under ultraviolet light (Fig. 6c). A calibration plot of the fluorescence intensity ratio $\left(I_{580} / I_{427}\right)$ against their corresponding UA concentrations was linear over the range of
Fig. 4 Fluorescence spectra (a) and UV-Vis spectra (b) of N-CDs interacting with various substances. Conditions: UA, $30 \mu \mathrm{M}$; uricase, $2.5 \mu \mathrm{g} \mathrm{mL}{ }^{-1}$; N-CDs, $12 \mu \mathrm{g} \mathrm{mL}^{-1}$; OPD, $3 \mathrm{mM}$; $\mathrm{I}^{-}$, $1.2 \mathrm{mM}$
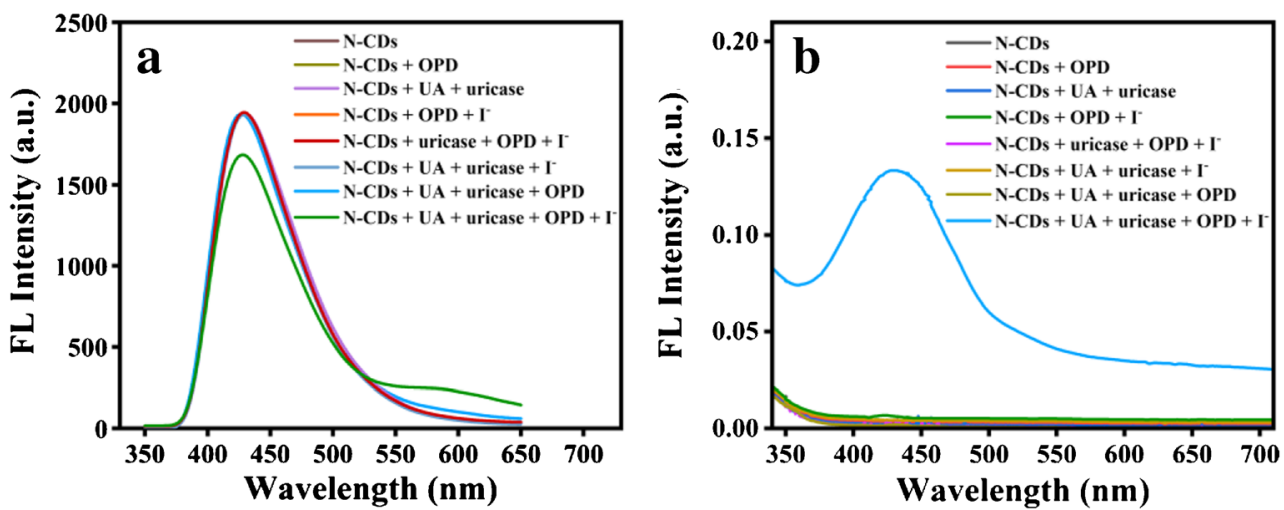
Fig. 5 Factors influencing the proposed sensor on $\mathbf{a} \mathrm{pH}, \mathbf{b}$ temperature, $\mathbf{c}$ time, $\mathbf{d}$ concentration of $\mathrm{I}^{-}$, and $\mathbf{e}$ concentration of OPD
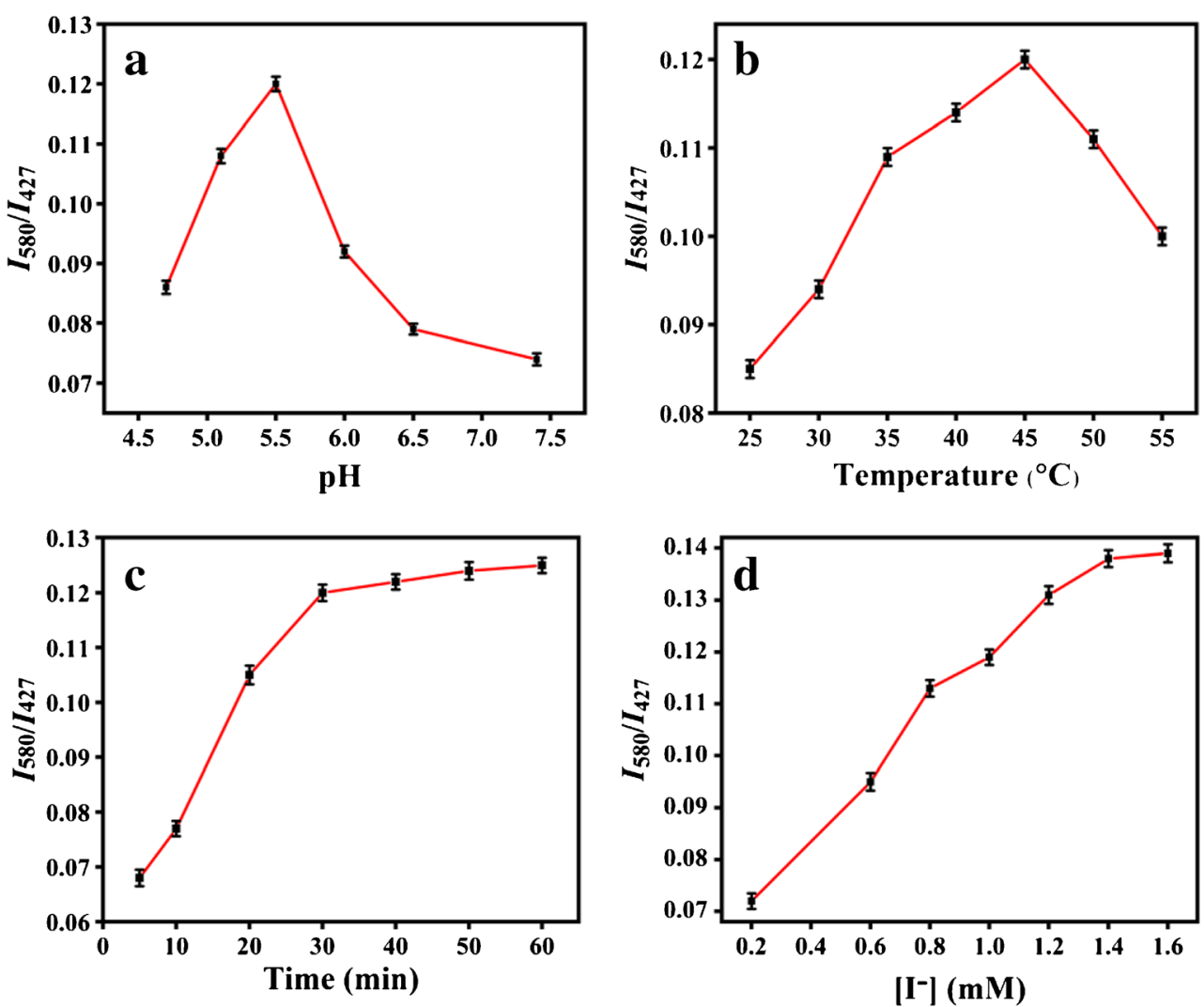

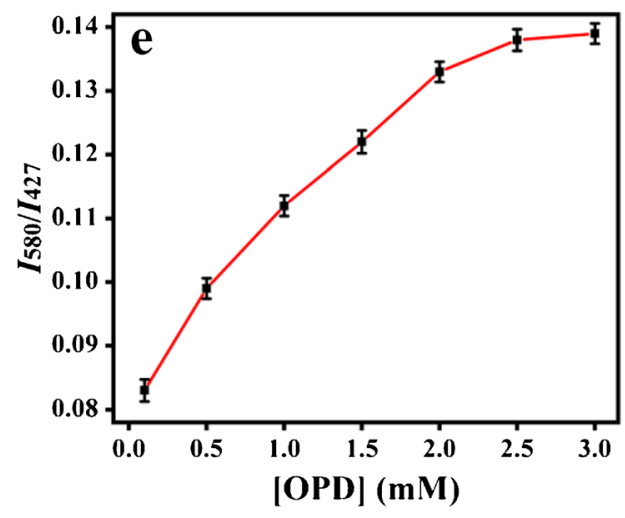

$0.5-150 \mu \mathrm{M}$. The resulting linear regression equation was $I_{580} / I_{427}=0.00182 \mathrm{C}_{\mathrm{UA}}+0.06937$, with a coefficient of determination $\left(R^{2}\right)$ of 0.992 (Fig. 6a, inset), demonstrating excellent linearity for quantitating UA in biological samples. Based on $3 \sigma / s$ (where $\sigma$ and $s$ represented the error of estimate and the slope of linear-regression-line, respectively), the limit of detection (LOD) was calculated to be $0.06 \mu \mathrm{M}$.

A calibration plot was also generated for the biosensor by absorption spectroscopy. The absorbance of the biosensor system at $427 \mathrm{~nm}$ increased with sequential additions of UA (Fig. 6a). The color of the solution gradually changed from colorless to yellow and was easily discernible. In the calibration curve (Fig. 6b, inset), there was an excellent linear relationship between $\Delta A\left(\Delta A=A-A_{0}\right.$, where $A$ represented the absorbance of the biosensor in the presence of $\mathrm{UA}$, and $A_{0}$ represented the absorbance in the absence of UA) and UA concentration over the range of 1-70 $\mu \mathrm{M}$. The resulting linear regression equation was $\Delta A=0.00279 \mathrm{C}_{\mathrm{UA}}$ +0.05907 , with an $R^{2}$ of 0.993 . The detection limit for the absorption spectroscopy assay was calculated to be $0.4 \mu \mathrm{M}$. In addition, we compared the sensor system with other biosensors reported in the literature, and the data are summarized in Table 1 [37-42]. Wang et al. built a colorimetric assay for detecting UA using $\mathrm{MoS}_{2}$ nanoflakes which can catalyze the oxidation of TMB by $\mathrm{H}_{2} \mathrm{O}_{2}$ [37]. Only colorimetric signals were used, and only serum samples were 
Fig. 6 Fluorescence emission spectra (a) excited at $330 \mathrm{~nm}$ and UV-Vis spectroscopy (b) of the proposed system for various UA concentrations (from low to high concentration: $0.5,1,2,2.5,5,10$, $20,30,50,70,100,150 \mu \mathrm{M})$, inset: the calibration curves. Color evolution for different UA concentrations under ultraviolet light (c) and under daylight (d)
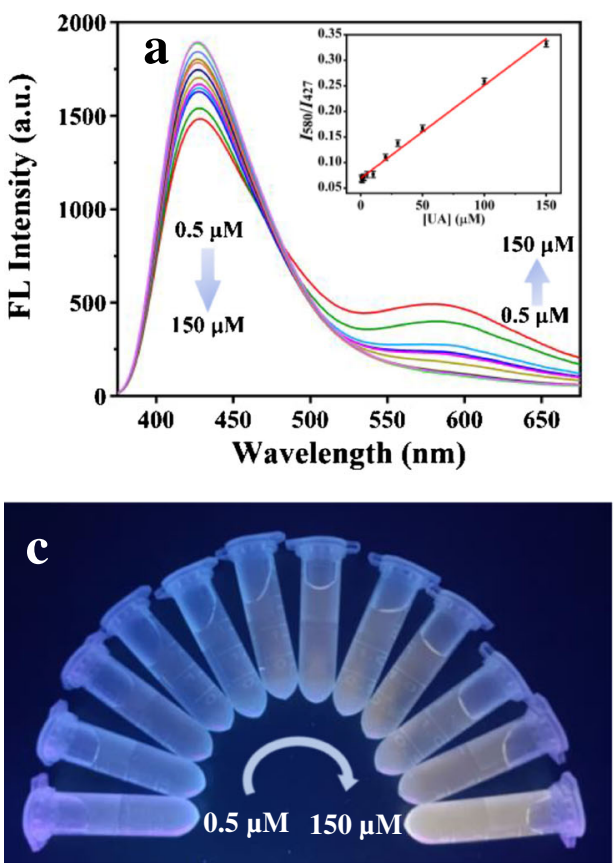
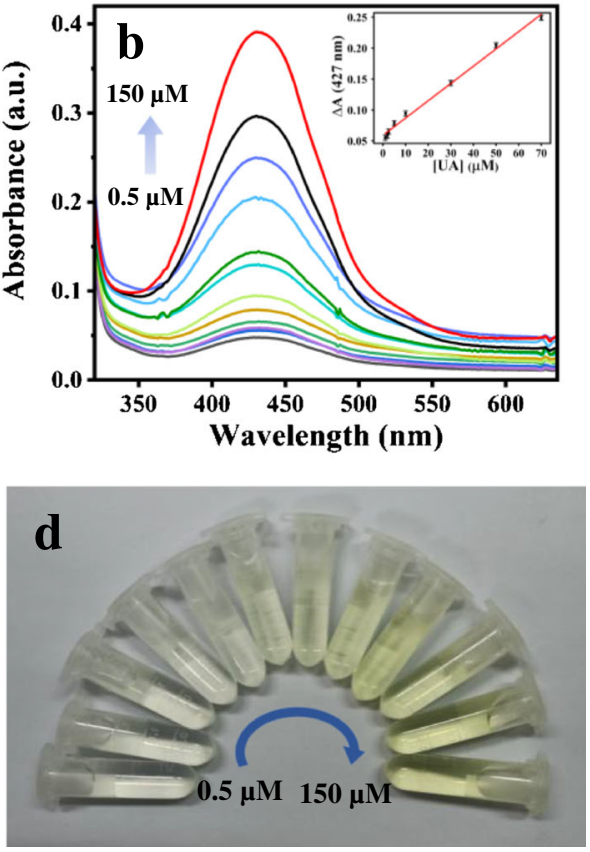

analyzed in the work. However, the proposed sensor in our work was dual-read for detecting UA and could be applied in serum and urine samples, which is relatively more diverse and reliable. Liu et al. reported the detection of UA by a ratiometric fluorescence probe based on the IFE between $\mathrm{Au} / \mathrm{Ag}$ NCs and DAP [40]. Herein, two noble metals were used and the LOD was $5.1 \mu \mathrm{M}$. In comparison, the sensor in this work has a low detection limit and a low material cost. Of note, the N-CDs-based sensor demonstrated excellent sensitivity for UA.

\section{The selectivity test of the sensor}

To evaluate the selectivity of the N-CDs-based sensor for quantitating UA concentrations, we investigated its response to a host of other potential interfering compounds in biological and clinical samples, including glucose (Glu), histidine (His), phenylalanine (Phe), creatinine (Cr), dopamine (DA), glutathione (GSH), urea, ascorbic acid (AA), and cysteine (Cys), as well three biologically relevant cations $\left(\mathrm{K}^{+}, \mathrm{Na}^{+}\right.$, and $\left.\mathrm{NH}_{4}{ }^{+}\right)$. Using the optimal experimental conditions determined previously, the biosensor was able to measure UA concentrations in the presence of individual interferences as well as a mixture of all of the interferences with UA. The normal concentration of AA in body fluids is far less than that of UA, especially when eating foods with low concentrations of ascorbic acid [43, 44]. In addition, the normal levels of UA in human urine and serum are far greater than the corresponding levels of cysteine [36]. Therefore, the concentrations of AA and Cys for the following experiment were chosen to be $7.5 \mu \mathrm{M}$ and $15 \mu \mathrm{M}$, respectively. As shown in Fig. 7a and 7b, only UA caused conspicuous changes in the biosensor system compared to the other substances tested individually. Then, different species were tested in
Table 1 Comparison of other determination methods for UA

\begin{tabular}{lllll}
\hline Detection method & Materials used & Linear range $(\mu \mathrm{M})$ & LOD $(\mu \mathrm{M})$ & Reference \\
\hline Colorimetry & $\mathrm{TMB}-\mathrm{MoS}_{2} / \mathrm{uricase}$ & $0.5-100$ & 0.3 & {$[37]$} \\
Electrochemistry & $\mathrm{Fc} @ \beta-\mathrm{CD} / \mathrm{N}-\mathrm{CD} / \mathrm{GCE}$ & $5-120$ & 0.08 & {$[38]$} \\
Colorimetry & $\mathrm{TMB}-\mathrm{CoP} / \mathrm{NF}$ & $1-200$ & 1.0 & {$[39]$} \\
Fluorescence & $\mathrm{BSA}-\mathrm{Au} / \mathrm{Ag} \mathrm{NCs}$ & $5-50$ & 5.1 & {$[40]$} \\
SERS & $\mathrm{AgNP} / \mathrm{ZnO} / \mathrm{Fe} 3 \mathrm{O} 4$ & $0.5-10$ & 0.365 & {$[8]$} \\
Fluorescence & $\mathrm{GSH}-\mathrm{Ti} \mathrm{C}_{2} \mathrm{MQDs}$ probe & $1.2-75$ & 0.12 & {$[41]$} \\
Electrochemistry & $\mathrm{MoS}_{2}$ based & $10-400$ & 1.169 & {$[42]$} \\
Fluorescence/colorimetric & $\mathrm{DAP} / \mathrm{N}-\mathrm{CD} /$ uricase/ $/$ & $0.5-150$ & 0.06 & This work \\
\hline
\end{tabular}




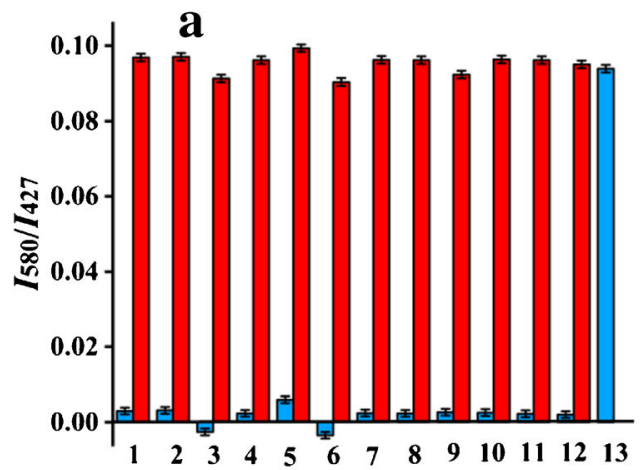

Fig. 7 The selectivity of the proposed sensor for fluorescence (a) and UV absorption (b) detection of UA. In the absence (blue column) and presence (red column) of UA, the interfering substances were added separately to the reaction systems. Each number corresponds to the following substances: (1) $\mathrm{NaCl}$, (2) $\mathrm{KNO}_{3}$, (3) $\mathrm{NH}_{4} \mathrm{Cl}$, (4) urea, (5)

the presence of UA, and the results showed no significant difference. The colors of the solutions containing the above reaction systems in the presence and absence of UA are shown in Fig. S1a and Fig. S1b. Based on these results, it was concluded that the dual-read assay for UA was highly selective and feasible for the quantitation of UA in human serum and urine samples.

\section{Application of the sensor in serum and urine}

Five serum and two urine samples were acquired to assess the practicality of dual-read sensing platform by this sensor. The results of the absorbance and fluorescence detection of UA were consistent with the results from the biochemistry analyzer, which are summarized in Tables 2 and 3. For the fluorescence determination, the recoveries were in the ranges of $94.6-103.3 \%$ and 99.6-105.8\% for the human serum and urine samples, respectively. For the absorption measurements, the recoveries were in the ranges of $99.3-102.9 \%$ and 90.1 $-95.0 \%$ for the human serum and urine samples, respectively. The relative standard deviation ( $\mathrm{RSD}, n=3)$ of

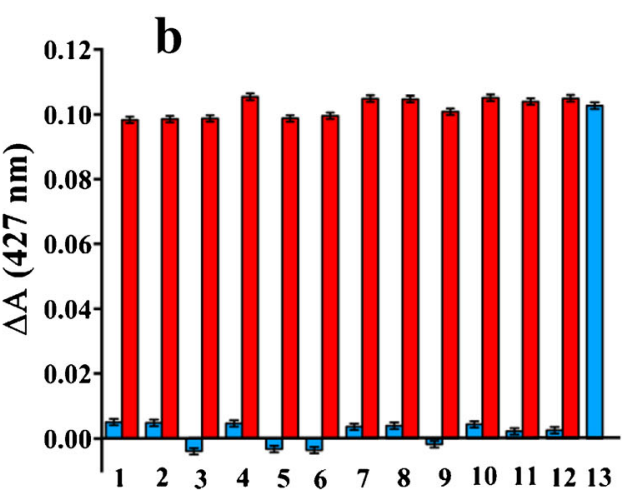

AA, (6) Cys, (7) Cr, (8) Glu, (9) DA, (10) GSH, (11) His, (12) Phe, and (13) UA. The concentration of UA and Cys was $15 \mu \mathrm{M}$, the concentration of AA was $7.5 \mu \mathrm{M}$, and the concentration of other interfering substances was $150 \mu \mathrm{M}$

the actual samples in the two detection modes was less than $3.6 \%$, which revealed that the dual-read detection of UA had excellent accuracy.

One advantage of colorimetric methods is that color changes can be observed visually. As shown in the images in S2a and S2c, the colors of both the serum and urine samples were exactly the same as the range of colors based on the UA concentrations shown in Fig. $6 \mathrm{~d}$. This was consistent with the calibration method performed by standard addition of UA to the biosensor system, as the colors of the serum and urine samples changed accordingly (S2b and S2d). Nonetheless, when we tested urine samples, it was found they exhibited a blue-green light emission at $430 \mathrm{~nm}$. Fortunately, the fluorescent background interference can be ignored until the sample was diluted to nearly 50-fold using PBS solution. The proposed assay is not suitable for samples with excessively strong fluorescent background. Thus, we concluded that the sensing strategy developed herein was practical and reliable for quantitation of UA in clinically relevant samples.

Table 2 Fluorescence determination of UA in human serum and urine samples $(n=3)$

\begin{tabular}{llllllll}
\hline Sample & Proposed sensor & $\begin{array}{l}\text { Biochemistry } \\
\text { analyzer }\end{array}$ & Average relative error (\%) & Added & Found & Recovery (\%) & R.S.D. (\%) \\
\hline Serum 1 & $305 \mu \mathrm{M}$ & $316 \mu \mathrm{M}$ & 3.3 & $400 \mu \mathrm{M}$ & $667 \mu \mathrm{M}$ & 94.6 & 2.3 \\
Serum 2 & $632 \mu \mathrm{M}$ & $601 \mu \mathrm{M}$ & & $400 \mu \mathrm{M}$ & $1066 \mu \mathrm{M}$ & 103.3 & 2.0 \\
Serum 3 & $554 \mu \mathrm{M}$ & $540 \mu \mathrm{M}$ & $484 \mu \mathrm{M}$ & $400 \mu \mathrm{M}$ & $943 \mu \mathrm{M}$ & 98.8 & 1.2 \\
Serum 4 & $493 \mu \mathrm{M}$ & $352 \mu \mathrm{M}$ & & $400 \mu \mathrm{M}$ & $889 \mu \mathrm{M}$ & 100.7 & 1.7 \\
Serum 5 & $341 \mu \mathrm{M}$ & - & $400 \mu \mathrm{M}$ & $752 \mu \mathrm{M}$ & 99.6 & 2.4 \\
Urine 1 & $3.04 \mathrm{mM}$ & - & $2.00 \mathrm{mM}$ & $5.17 \mathrm{mM}$ & 102.8 & 3.5 \\
Urine 2 & $1.74 \mathrm{mM}$ & - & $2.00 \mathrm{mM}$ & $3.84 \mathrm{mM}$ & 105.8 & 3.2 \\
\hline
\end{tabular}


Table 3 Colorimetry determination of UA in human serum and urine samples $(n=3)$

\begin{tabular}{llllllll}
\hline Sample & Proposed sensor & $\begin{array}{c}\text { Biochemistry } \\
\text { analyzer }\end{array}$ & Average relative error (\%) & Added & Found & Recovery (\%) & R.S.D. (\%) \\
\hline Serum 1 & $327 \mu \mathrm{M}$ & $316 \mu \mathrm{M}$ & 3.7 & $400 \mu \mathrm{M}$ & $748 \mu \mathrm{M}$ & 102.9 & 1.9 \\
Serum 2 & $619 \mu \mathrm{M}$ & $601 \mu \mathrm{M}$ & & $400 \mu \mathrm{M}$ & $1026 \mu \mathrm{M}$ & 99.3 & 2.3 \\
Serum 3 & $563 \mu \mathrm{M}$ & $540 \mu \mathrm{M}$ & $484 \mu \mathrm{M}$ & $400 \mu \mathrm{M}$ & $938 \mu \mathrm{M}$ & 97.4 & 2.8 \\
Serum 4 & $467 \mu \mathrm{M}$ & $352 \mu \mathrm{M}$ & & $400 \mu \mathrm{M}$ & $873 \mu \mathrm{M}$ & 100.7 & 1.5 \\
Serum 5 & $365 \mu \mathrm{M}$ & - & $400 \mu \mathrm{M}$ & $752 \mu \mathrm{M}$ & 98.3 & 1.7 \\
Urine 1 & $2.87 \mathrm{mM}$ & - & $2.00 \mathrm{mM}$ & $4.39 \mathrm{mM}$ & 90.1 & 3.1 \\
Urine 2 & $1.63 \mathrm{mM}$ & - & $2.00 \mathrm{mM}$ & $3.45 \mathrm{mM}$ & 95.0 & 2.6 \\
\hline
\end{tabular}

\section{Conclusion}

A ratiometric fluorescence and colorimetric dual-read assay for UA based on nitrogen-doped carbon dots (N-CDs) was successfully designed and applied in the measurement of UA in human serum and urine samples. The N-CDs did not require complex synthetic techniques, which is advantageous for their applications and scalability. Both ratiometric fluorescence and colorimetric signals generated by the N-CDs/ uricase $/ \mathrm{I}^{-}$probe were used for the quantitative analysis of UA. The colorimetric shifts upon the interactions of the biosensor with varying concentrations of UA levels were observed visually, which made it easy to determine the relative concentration of UA by eye; the concentrations were further determined by the absorbance values using the calibration plot. Compared to traditional colorimetric detection methods, horseradish peroxidase was replaced with iodide, which greatly reduced the risk of inactivity and cost. Overall, the prepared N-CDs exhibited excellent optical properties and stability, and the dual-read assay for quantitating UA demonstrated excellent sensitivity, selectivity, and a considerable potential for derivatization to meet the needs of various practical applications.

Supplementary Information The online version contains supplementary material available at https://doi.org/10.1007/s00604-021-04971-2.

Declarations The study was approved by the ethics committee of Jiangxi Medical College, and their guidelines were followed throughout this study. The serum samples involved in our research were from individuals with gout. Informed consent was obtained from all human participants.

Competing interests The authors declare no competing interests.

Funding This work was financially supported by the National Natural Science Foundation of China $(21765015,21808099)$ and the Science and Technology Innovation Platform of Jiangxi Province (20192BCD40001), China.

\section{Compliance with ethical standards}

Conflict of interest The authors declare that they have no competing of interests.

\section{References}

1. Wang Q, Wen X, Kong J (2020) Recent progress on uric acid detection: a review. Crit Rev Anal Chem 50:359-375. https://doi. org/10.1080/10408347.2019.1637711

2. Wang X, Li Z, Lai J, Tang X, Qiu P (2018) Sensitive and highly selective biosensor based on triangular Au nanoplates for detection of uric acid in human serum. Chem Afr 1:29-35. https://doi.org/10. 1007/s42250-018-0001-0

3. Wu D, Lu H, Xie H, Wu J, Wang C, Zhang Q (2015) Uricasestimulated etching of silver nanoprisms for highly selective and sensitive colorimetric detection of uric acid in human serum. Sensors Actuators B Chem 221:1433-1440. https://doi.org/10. 1016/j.snb.2015.07.088

4. Galassi F, Borghi C (2015) A brief history of uric acid: from gout to cardiovascular risk factor. Eur J Intern Med 26:373. https://doi.org/ 10.1016/j.ejim.2015.04.005

5. Ma L, Wei L, Chen H, Zhang Z, Yu Q, Ji Z, Jiang L (2016) Influence of urate-lowering therapies on renal handling of uric acid. Clin Rheumatol 35:133-141. https://doi.org/10.1007/s10067-0142806-9

6. Perticone M, Tripepi G, Maio R, Cimellaro A, Addesi D, Baggetta R, Sciacqua A, Sesti G, Perticone F (2017) Risk reclassification ability of uric acid for cardiovascular outcomes in essential hypertension. Int J Cardiol 243:473-478. https://doi.org/10.1016/j.ijcard. 2017.05.051

7. Shi B, Su Y, Duan Y, Chen S, Zuo W (2019) A nanocomposite prepared from copper(II) and nitrogen-doped graphene quantum dots with peroxidase mimicking properties for chemiluminescent determination of uric acid. Microchim Acta 186:1-10. https://doi. org/10.1007/s00604-019-3491-9

8. Misra N, Kumar V, Borde L, Varshney L (2013) Localized surface plasmon resonance-optical sensors based on radiolytically synthesized silver nanoparticles for estimation of uric acid. Sensors Actuators B Chem 178:371-378. https://doi.org/10.1016/j.snb. 2012.12.110

9. Li Z, Wu M, Yao J, Guo J, Liao X, Song S, Li J, Duan G, Zhou Y, Wu X (2020) Caution on kidney dysfunctions of COVID-19 patients. Lancet Infect Dis:1-38. https://doi.org/10.2139/ssrn. 3559601 
10. Khouchlaa A, Bouyahya A (2020) Covid-19 nephropathy: probable mechanisms of kidney failure. J Nephropathol 9:35. https://doi.org/ 10.34172/jnp.2020.35

11. Li Q, Qiu Y, Han W, Zheng Y, Wang X, Xiao D, Mao M, Li Q (2018) Determination of uric acid in biological samples by high performance liquid chromatography-electrospray ionizationtandem mass spectrometry and study on pathogenesis of pulmonary arterial hypertension in pulmonary artery endothelium cells. RSC Adv 8:25808-25814. https://doi.org/10.1039/ C7RA12702B

12. Azmi N, Ramli N, Abdullah J, Hamid M, Sidek H, Abd Rahman S, Ariffin N, Yusof N (2015) A simple and sensitive fluorescence based biosensor for the determination of uric acid using $\mathrm{H}_{2} \mathrm{O}_{2}$-sensitive quantum dots/dual enzymes. Biosens Bioelectron 67:129133. https://doi.org/10.1016/j.bios.2014.07.056

13. Herrasti Z, Martínez F, Baldrich E (2016) Detection of uric acid at reversibly nanostructured thin-film microelectrodes. Sensors Actuators B Chem 234:667-673. https://doi.org/10.1016/j.snb. 2016.05.018

14. Norazmi N, Abdul Rasad Z, Mohamad M, Manap H (2017) Uric acid detection using uv-vis spectrometer. Conf Ser: Mater Sci Eng 257:012031-012037. https://doi.org/10.1088/1757-899X/257/1/ 012031

15. Lu J, Xiong Y, Liao C, Ye F (2015) Colorimetric detection of uric acid in human urine and serum based on peroxidase mimetic activity of MIL-53(Fe). Anal Methods 7:9894-9899. https://doi.org/10. 1039/c5ay02240a

16. Yao D, Vlessidis A, Evmiridis N (2003) Microdialysis sampling and monitoring of uric acid in vivo by a chemiluminescence reaction and an enzyme on immobilized chitosan support membrane. Anal Chim Acta 478:23-30. https://doi.org/10.1016/S00032670(02)01484-8

17. Melisew T, Peter L, Liu B, Dirk W, Jyisy Y, Hendrik S (2019) Preparation of silver nanoparticles coated $\mathrm{ZnO} / \mathrm{Fe}_{3} \mathrm{O}_{4}$ composites using chemical reduction method for sensitive detection of uric acid via surface-enhanced Raman spectroscopy. Anal Chim Acta 1073: 62-71. https://doi.org/10.1016/j.aca.2019.04.061

18. Wang Y, Yang Y, Liu W, Ding F, Zou P, Wang X, Zhao Q, Rao H (2019) A carbon dot-based ratiometric fluorometric and colorimetric method for determination of ascorbic acid and of the activity of ascorbic acid oxidase. Microchim Acta 186:1-10. https://doi.org/ 10.1007/s00604-019-3341-9

19. Pei X, Pan Y, Zhang L, Lv Y (2020) Recent advances in ratiometric luminescence sensors. Appl Spectrosc Rev 56:324-345. https://doi. org/10.1080/05704928.2020.1793770

20. Miao S, Liang K, Kong B (2020) Förster resonance energy transfer (FRET) paired carbon dot-based complex nanoprobes: versatile platforms for sensing and imaging applications. Mater Chem Front 4:128-139. https://doi.org/10.1039/c9qm00538b

21. Shangguan J, Huang J, He D, He X, Wang K, Ye R, Yang X, Qing $\mathrm{T}$, Tang J (2017) Highly $\mathrm{Fe}^{3+}$-selective fluorescent nanoprobe based on ultrabright N/P codoped carbon dots and its application in biological samples. Anal Chem 89:7477-7484. https://doi.org/ 10.1021/acs.analchem.7b01053

22. Matai I, Sachdev A, Gopinath P (2015) Self-assembled hybrids of fluorescent carbon dots and PAMAM dendrimers for epirubicin delivery and intracellular imaging. ACS Appl Mater Interfaces 7: 11423-11435. https://doi.org/10.1021/acsami.5b02095

23. Feng J, Wang W, Hai X, Yu Y, Wang J (2016) Green preparation of nitrogen-doped carbon dots derived from silkworm chrysalis for cell imaging. J Mater Chem B 4:387-393. https://doi.org/10.1039/ C5TB01999K

24. Guo X, Wang C, Yu Z, Chen L, Chen S (2012) Facile access to versatile fluorescent carbon dots toward light-emitting diodes. Chem Commun 48:2692-2694. https://doi.org/10.1039/ c2cc17769b
25. Barman M, Jana B, Bhattacharyya S, Patra A (2014) Photophysical properties of doped carbon dots $(\mathrm{N}, \mathrm{P}$, and $\mathrm{B})$ and their influence on electron/hole transfer in carbon dots-nickel (II) phthalocyanine conjugates. J Phys Chem C 118:20034-20041. https://doi.org/10. 1021/jp507080c

26. Liu Y, Liao M, He X, Liu X, Kou X, Xiao D (2015) One-step synthesis of highly luminescent nitrogen-doped carbon dots for selective and sensitive detection of mercury (II) ions and cellular imaging. Anal Sci 31:971-977. https://doi.org/10.2116/analsci.31. 971

27. Ni P, Sun Y, Dai H, Jiang S, Lu W, Wang Y, Li Z, Li Z (2016) Colorimetric determination of the activity of acetylcholinesterase and its inhibitors by exploiting the iodide-catalyzed oxidation of 3,3',5,5'-tetramethylbenzidine by hydrogen peroxide. Microchim Acta 183:2589-2595. https://doi.org/10.1007/s00604-016-1874-8

28. Wu C, Zhu L, Lu Q, Li H, Zhang Y, Yao S (2019) A dual-signal colorimetric and ratiometric fluorescent nanoprobe for enzymatic determination of uric acid by using silicon nanoparticles. Microchim Acta 186:1-8. https://doi.org/10.1007/s00604-0193862-2

29. Wang X, Chen S, Tang X, Lin D, Qiu P (2019) Ultrasensitive detection of uric acid in serum of patients with gout by a new assay based on Pt@Ag nanoflowers. RSC Adv 9:36578-36585. https:// doi.org/10.1039/c9ra06481h

30. Ma Y, Cen Y, Sohail M, Xu G, Wei F, Shi M, Xu X, Song Y, Ma Y, $\mathrm{Hu} \mathrm{Q}$ (2017) A ratiometric fluorescence universal platform based on $\mathrm{N}, \mathrm{Cu}$ codoped carbon dots to detect metabolites participating in $\mathrm{H}_{2} \mathrm{O}_{2}$-generation reactions. ACS Appl Mater Interfaces 9:3301133019. https://doi.org/10.1021/acsami.7b10548

31. Dong Y, Dong H, Yang HB, Guo C, Shao J, Chi Y, Li CM, Yu T (2013) Carbon-based dots co-doped with nitrogen and sulfur for high quantum yield and excitation-in-dependent emission. Angew Chem Int Ed 52:7800-7804. https://doi.org/10.1002/anie. 201301114

32. Zhang WJ, Liu SG, Han L, Luo HQ, Li NB (2019) A ratiometric fluorescent and colorimetric dual-signal sensing platform based on $\mathrm{N}$-doped carbon dots for selective and sensitive detection of copper (II) and pyrophosphate ion. Sensors Actuators B Chem 283:215221. https://doi.org/10.1016/j.snb.2018.12.012

33. Wang L, Liu Y, Yang Z, Wang Y, Rao H, Yue G, Wu C, Lu C, Wang $X$ (2020) A ratiometric fluorescence and colorimetric dualmode assay for $\mathrm{H}_{2} \mathrm{O}_{2}$ and xanthine based on $\mathrm{Fe}, \mathrm{N}$ co-doped carbon dots. Dyes Pigments 180:108486. https://doi.org/10.1016/j.dyepig. 2020.108486

34. Chen H, Lu Q, He K, Liu M, Zhang Y, Yao S (2018) A cyclic signal amplification strategy to fluorescence and colorimetric dualreadout assay for the detection of $\mathrm{H}_{2} \mathrm{O}_{2}$-related analytes and application to colorimetric logic gate. Sensors Actuators B Chem 260: 908-917. https://doi.org/10.1016/j.snb.2018.01.085

35. Mahler HR, Baum HM, Hübscher G (1956) Enzymatic oxidation of urate. Science 124:705-708. https://doi.org/10.1126/science.124. 3225.705

36. Li F, He T, Wu S, Peng Z, Qiu P, Tang X (2021) Visual and colorimetric detection of uric acid in human serum and urine using chitosan stabilized gold nanoparticles. Microchem J 164:105987. https://doi.org/10.1016/j.microc.2021.105987

37. Wang X, Yao Q, Tang X, Zhong H, Qiu P (2019) A highly selective and sensitive colorimetric detection of uric acid in human serum based on $\mathrm{MoS}_{2}$-catalyzed oxidation TMB. Anal Bioanal Chem 411: 943-952. https://doi.org/10.1007/s00216-018-1524-6

38. Zhang H, Dai P, Huang L, Huang Y, Huang Q, Zhang W, Wei C, Hu S (2014) A nitrogen-doped carbon dot/ferrocene@ $\beta$-cyclodextrin composite as an enhanced material for sensitive and selective determination of uric acid. Anal Methods 6:2687-2691. https://doi. org/10.1039/C4AY00140K 
39. He Y, Qi F, Niu X, Zhang W, Zhang X, Pan J (2018) Uricase-free on-demand colorimetric biosensing of uric acid enabled by integrated $\mathrm{CoP}$ nanosheet arrays as a monolithic peroxidase mimic. Anal Chim Acta 1021:113-120. https://doi.org/10.1016/j.aca.2018.02. 073

40. Wang X, Zhu G, Cao W, Liu Z, Pan C, Hu W, Zhao W, Sun J (2019) A novel ratiometric fluorescent probe for the detection of uric acid in human blood based on $\mathrm{H}_{2} \mathrm{O}_{2}$-mediated fluorescence quenching of gold/silver nanoclusters. Talanta 191:46-53. https:// doi.org/10.1016/j.talanta.2018.08.015

41. Liu M, He Y, Zhou J, Ge Y, Zhou J, Song G (2020) A "naked-eye" colorimetric and ratiometric fluorescence probe for uric acid based on $\mathrm{Ti}_{3} \mathrm{C}_{2}$ MXene quantum dots. Anal Chim Acta 1103:134-142. https://doi.org/10.1016/j.aca.2019.12.069

42. Sha R, Vishnu N, Badhulika S (2019) $\mathrm{MoS}_{2}$ based ultra-low-cost, flexible, non-enzymatic and non-invasive electrochemical sensor for highly selective detection of Uric acid in human urine samples. Sensors Actuators B Chem 279:53-60. https://doi.org/10.1016/j. snb.2018.09.106

43. Wang YH, Dhariwal KR, Levine M (1992) Ascorbic acid bioavailability in humans: ascorbic acid in plasma, serum, and urine. Ann N Y Acad Sci 669:383-386. https://doi.org/10.1111/j.1749-6632. 1992.tb17130.x

44. Simon JA, Hudes ES, Browner WS (1998) Serum ascorbic acid and cardiovascular disease prevalence in US adults. Epidemiology 9: 316-321. https://doi.org/10.1097/00001648-199805000-00017

Publisher's note Springer Nature remains neutral with regard to jurisdictional claims in published maps and institutional affiliations. 